\title{
Use of fractals to measure anisotropy in point patterns extracted with the DPT of an image
}

\author{
I. Fabris-Rotelli ${ }^{\mathrm{a}, *}$, A. Stein ${ }^{\mathrm{a}, \mathrm{b}}$ \\ a University of Pretoria, South Africa \\ ${ }^{\mathrm{b}}$ University of Twente, The Netherlands
}

\section{A R T I C L E I N F O}

\section{Article history:}

Received 14 November 2019

Received in revised form 26 April 2020

Accepted 10 May 2020

Available online 26 May 2020

\section{Keywords:}

Discrete pulse transform

Fractal

$h t$-index

Anisotropy

Spatial point pattern

Texture image

\begin{abstract}
A B S T R A C T
Images are particular and well-known instances of spatial big data. Typically spatial data are scale specific and in this paper, we propose mechanisms to effectively address issues of scale in the analysis of images. We focus on spatial data extracted from images using the Discrete Pulse Transform (DPT). The DPT extracts discrete pulses from images at multiple scales that are recognisable as connected components. Traditionally, fractals are used for this purpose, but they fall short as the process underlying fractality is usually either absent or poorly understood. This paper investigates the $H t$-index (head/tail break) as an alternative, merging ideas from image analysis and spatial statistics. More specifically, we use the $\mathrm{Ht}$-index for the analysis of anisotropic point patterns that are obtained from applying the DPT. We propose a multi-level $\mathrm{Ht}$-index decomposition in this regard. This is the first mechanism for the DPT enabling an informed partition of the scale-space. The results show that the $H t$-index is well suited to identify the anisotropic structure location within specific scales and thereby substantially reduces computational costs. We conclude that the use of the $\mathrm{Ht}$-index is promising and is well-suited for the further analysis of spatial big data.
\end{abstract}

(c) 2020 Elsevier B.V. All rights reserved.

\section{Introduction}

Big spatial data show computational complications, as they are more intense than non-spatial big data. The analysis in such a case still requires attention. Here we focus on spatial data extracted

\footnotetext{
* Corresponding author.

E-mail address: inger.fabris-rotelli@up.ac.za (I. Fabris-Rotelli).
} 
from images by using the Discrete Pulse Transform (DPT) (Fabris-Rotelli and Stein, 2018). The DPT extracts discrete pulses, recognisable as connected components, from images at multiple scales. The scale is measured as the number of pixels. Scaling is referred to in a geographical sense as a universal form or pattern occurring across scales (Jiang and Sui, 2014). These DPT pulses at various scales characterise the texture of an image and can be effectively spatially modelled as point patterns, as shown in Fabris-Rotelli and Stein (2018). The size of the resulting point pattern presents big spatial data having in the 100 000's points for a single band image. Texture knowledge is useful for remote sensing classification applications, for example, but further adds complexity to big spatial data due to the presence of multiple scales, resolution and multivariate data. We are specifically interested here in determining the anisotropy of such a point process with the view of picking up directionality in an image for texture analysis. Al-Saidi and AbdulWahed (2018) define an image texture as "a structure that preserves some statistical properties in the colour or brightness distribution and repeatedly makes patterns". Fazel-Rezai and Kinsner (1999) state that texture provides essential structure information of an image and Roux et al. (2013) comment that self-similar processes with anisotropy model image texture processes well. In the past, the fractal dimension has been investigated in image analysis, specifically texture analysis. The theoretical development has increased as computing grows (Chen et al., 1993; Ida and Sambonsugi, 1998; Kisan et al., 2016; Lam and Li, 2010; Liu, 2008; Melnikov, 2007; Rigaut et al., 1998; Rosen, 1995; Wang et al., 2011; Zhao and Liu, 2005). It has been used in remote sensing (Al-Saidi and Abdul-Wahed, 2018; Berizzi et al., 2001; Chenoweth et al., 1995; Lam, 1990; Zhu and Yang, 2010; Di Martino et al., 2010; Riccio et al., 2014; Sawada et al., 2001), image inpainting (Xiu-hong and Bao-long, 2009; Bai et al., 2011), image matching with texture (Dolez and Vincent, 2007), denoising (Ghazel et al., 2003; Malviya, 2008), restoration (Hamano et al., 1996), segmentation (Ida and Sambonsugi, 1998), compression (Ismail et al., 2010; Jiang, 1995), shape classification and segmentation (Kisan et al., 2016; Nayak et al., 2015), interpolation (Shi et al., 2008), classification (Shih, 2008), superresolution (Wee and Shin, 2010), medical imaging (Hong and Huidong, 2012; Priya et al., 2011; Qi et al., 2009; Tang and Wang, 2006) and specifically in texture analysis (Avadhanam and Mitra, 1994; Costa et al., 2012; Fazel-Rezai and Kinsner, 1999). Wavelet based techniques are also widely used and are powerful in image processing techniques. They have been used in directional texture analysis in Kumar (1995), Po and Do (2006), Miya and Aghagotza (2004), Zhang et al. (2016a), Nicolis et al. (2011) and Roux et al. (2013). The DPT provides an alternative multiscale method, which has a number of advantages. It provides an already discrete approach to extracting multiscale information from images. Further, it has strong mathematical properties such as the use of non-linear idempotent operators with a semi-group structure, edgepreservation (non-blurring) and structure consistency with a perfect reconstruction obtained via a simple sum. The novelty of this paper is that we merge the idea of directional image analysis with spatial analysis of point patterns, providing also the first method to extract important scales of the scale-space built by the DPT.

We now turn to identifying the fractal structure of the DPT. A set or pattern is said to be fractal if there are far more small pattern elements than larger or, similarly, the scaling pattern of this abundant small pattern elements compared to large occurs numerous times (Li et al., 2016). Fractal geometry (Mandelbrot, 1967, 1982) provides a geometry for rough and irregular forms, including a quantitative value known as the fractal dimension. The fractal dimension, however, requires strict self-similarity through a power law that is seldom present in data with heavier tails. To overcome this problem, in this paper we investigate the $H t$-index as an alternative fractal measure in images, merging ideas from image analysis and spatial statistics. The $H t$-index (head/tails break) (Jiang et al., 2013) provides an effective method to visualise the hierarchy of a complex system. It measures the fractal nature of geographical data, as an alternative to the global natural breaks classification (Jenks, 1963), which minimises intraclass variance but is unable to capture scaling structure. The $\mathrm{Ht}$-index allows for any functional form and captures geostatistical data more appropriately (Jiang and Yin, 2014). It is defined as the number of repetitions for which the following is observed: the mean scale thresholds the data so that the majority ( $>50 \%$ ) of data values fall to the left of the mean scale. The subsequent repetition is conducting on only the data in the smaller tail. Larger complexity is visible with more repetitions measured. The Ht-index captures the idea of having many more 
small elements than large elements. It has been applied to city sizes, streets, population densities on lattice data, night light imagery pixel values, terrain surface DEM values and the evaluation of natural cities (Jiang and Sui, 2014; Jiang, 2013; Jiang and Miao, 2015), to name a few areas.

A DPT pattern can be seen as a collection of points, where the points all have a fractal shape. We note for instance that small point patterns are more naturally isotropic and stationary (Rajala et al., 2018). Hence, stationarity and isotropy of the DPT should be checked carefully. This can be complicated in the light of the big data nature of the DPT. Simply sampling the point pattern to reduce size is not effective. Ratcliffe (2004) shows that a $85 \%$ sample is needed to maintain the structure of a point pattern. This size sample does not alleviate the big data aspect. We thus make use of the $H t$-index as a fractal measure for spatial data from images to extract the true texture structure in a knowledgeable way. Li et al. (2016) state that the next step using the $H t$-index is to develop an understanding of the fractal structure. We do so here for point patterns extracted from texture images using the DPT. Jiang and Sui (2014) state that "scaling, if visualised properly using head/tail breaks can evoke a sense of beauty". We decompose an image to demonstrate this visualisation.

The objective of this paper is to use the $H t$-index for the analysis of point patterns that are obtained from applying the DPT on anisotropic images. The paper is structured as follows. Section 2 considers images in the context of big spatial data. Section 3 describes the extraction of a texture point pattern using the DPT, the employment of the $H t$-index to this point pattern and then anisotropic analysis. Section 4 applies the ideas to a texture image from the Brodatz texture database. ${ }^{1}$ Section 5 discusses the results and Section 6 concludes and suggests further avenues.

\section{Images as big spatial data}

Big spatial data (BSD) have an even larger impact computationally than traditional big data. In particular, spatial autocorrelation (Vatsavai et al., 2012), non-stationarity and anisotropy have to be considered. These properties are defined in terms of distances between pairs of points, hence BSD are more computationally intensive than non-spatial big data (Shekhar et al., 2011) and have further challenges as discussed in Miller and Goodchild (2015). There have been a number of books on BSD recently published (Evans et al., 2014, 2018; Shekhar and Jiang, 2017). A number of discussions on BSD and avenues to investigate can be found in Li et al. (2016), Cugler et al. (2013), Goodchild (2013) and Vatsavai et al. (2012). A shift to data-driven research and non-iterative algorithms, the need for improved spatial indexing, improved visualisation informed by human cognition and quality assurance are highlighted.

Remote sensing image analysis has emerged in the past decades and big data for this analysis has been explosive due to location-aware software. With the volume of data increasing with varying format the big data aspect frequently starts discussion. Big spatial data provide valuable information for more informative decision making. It has a huge potential in society such as water management, agriculture, transportation and public health (Lopez et al., 2014; Li et al., 2016). Smarter computing has been investigated to deal with BSD such as cluster computing (Yu et al., 2016), Hadoop-GIS for spatial querying (Chen et al., 2014), Hadoop and MapReduce (Jardak et al., 2014; Li et al., 2014), development of a spatial cumulative sum algorithm (Manogaran and Lopez, 2018), GPU usage (Zhang et al., 2017), cloud computing (Zhang et al., 2016b) and Java and Apache Spark (Wang et al., 2017).

\section{Methodology}

The DPT is a nonlinear multiscale decomposition of an image obtained through recursive application of the operators $L_{n}$ and $U_{n}$ (Anguelov and Fabris-Rotelli, 2010). The Discrete Pulse Transform (DPT) is obtained via the sequential application of the LULU operators $L_{n}$ and $U_{n}, n=1,2, \ldots, N$

\footnotetext{
1 http://www.ux.uis.no/tranden/brodatz.html
} 
where $N$ is the total number of pixels in the image. They are defined for $f \in \mathcal{A}\left(\mathbb{Z}^{2}\right)$, a vector lattice, and $n \in \mathbb{N}$, as

$$
\begin{aligned}
L_{n}(f)(x) & =\max _{V \in \mathcal{N}_{n}(x)} \min _{y \in V} f(y), x \in \mathbb{Z}^{2}, \\
U_{n}(f)(x) & =\min _{V \in \mathcal{N}_{n}(x)} \max _{y \in V} f(y), x \in \mathbb{Z}^{2},
\end{aligned}
$$

where $\mathcal{N}_{n}(x)=\{V \in \mathcal{C}: x \in V, \operatorname{card}(V)=n+1\}$ and $\mathcal{C}$ is a connection (see Fabris-Rotelli and Stein, 2018 for details not repeated here). The well-known concepts of 4- and 8-connectivity within pixel lattices are examples of mathematical connections. We make use of 4-connectivity for the DPT here, however the LULU operators are defined on any chosen connection satisfying the required connectivity properties.

The DPT is obtained via iterative application of the operators $L_{n}, U_{n}$ with $n$ increasing from 1 to $N$, decomposing the image into a number of pulses $\phi_{n s}, s=1, \ldots, \gamma(n)$, where $n$ is the scale (number of pixels in the connected component) and $s=1, \ldots, \gamma(n)$ the number of pulses at scale $n$, using the operations $P_{n}=L_{n} \circ U_{n}$ or $P_{n}=U_{n} \circ L_{n}$ and then $Q_{n}=P_{n} \circ P_{n-1} \circ \ldots \circ P_{1}$. The number of pulses at each scale $n$ varies and is represented as $\gamma(n)$ to clarify this. A pulse $\phi_{n s}$ is defined on a connected set $V \subset \mathbb{Z}^{2}$ if it has value $c$ (some constant) for $x \in V$ and value 0 otherwise. At each iteration the portions of the image which are filtered out by the application of $P_{n}, n=1,2, \ldots, N$ i.e. $\left(I-P_{n}\right)(f)=D_{n}(f)$, are retained until we obtain $Q_{N}(f)$, a constant function, where $I$ is the identity operator. The function $f$ is then decomposed as the DPT of $f$ :

$$
f=\sum_{n=1}^{N} D_{n}(f)=\sum_{n=1}^{N} \sum_{s=1}^{\gamma(n)} \phi_{n s}
$$

into pulses $\phi_{n s}$ (Fabris-Rotelli and Stein, 2018). The maximum scale is $N$ which is the total number of pixels in the image being considered. Further details are shown in Fabris-Rotelli and Stein (2018) and Anguelov and Fabris-Rotelli (2010). This image decomposition provides us with information about the structure content of the image at all scales from $n=1$ up to $N$.

We provide a short example to illustrate. Consider a tiny image

$$
f=\left[\begin{array}{lll}
2 & 1 & 3 \\
5 & 4 & 3
\end{array}\right] \text {. }
$$

The DPT proceeds as follows, sequentially removing local maximum and minimum sets,

$$
\begin{aligned}
L_{1}(f) & =\left[\begin{array}{lll}
2 & 1 & 3 \\
4 & 4 & 3
\end{array}\right] \\
U_{1} L_{1}(f) & =\left[\begin{array}{lll}
2 & 2 & 3 \\
4 & 4 & 3
\end{array}\right] \\
L_{2} U_{1} L_{1}(f) & =\left[\begin{array}{lll}
2 & 2 & 3 \\
3 & 3 & 3
\end{array}\right] \\
U_{2} L_{2} U_{1} L_{1}(f) & =\left[\begin{array}{lll}
3 & 3 & 3 \\
3 & 3 & 3
\end{array}\right]
\end{aligned}
$$

so that $f$ is decomposed as the connected portions removed at each filtering

$$
f=\left[\begin{array}{lll}
0 & 0 & 0 \\
1 & 0 & 0
\end{array}\right]+\left[\begin{array}{ccc}
0 & -1 & 0 \\
0 & 0 & 0
\end{array}\right]+\left[\begin{array}{lll}
0 & 0 & 0 \\
1 & 1 & 0
\end{array}\right]+\left[\begin{array}{ccc}
-1 & -1 & 0 \\
0 & 0 & 0
\end{array}\right]+\left[\begin{array}{lll}
3 & 3 & 3 \\
3 & 3 & 3
\end{array}\right] .
$$

As is seen in the example, due to the multiscale nature each pixel can belong to multiple pulses at different scales.

We focus on the pulses as spatial units and apply the $H t$-index to them. The number of pulses is huge presenting BSD. The use of the $H t$-index on this type of data provides informative fractal levels. The Ht-index is defined as one plus the recurring times for which there are more small structures than large, that is, it equals $h$ if this recurs $h-1$ times (Jiang, 2013). The procedure for calculation 
of the Ht-index is as a first step to determine the mean scale of all the structures. The number of structures smaller than this mean, $n_{\text {less }}$, and the number of structures larger than this mean, $n_{\text {more }}$, are calculated. If $n_{\text {less }}>n_{\text {more }}$, the second step is to repeat this process. The mean of the tail (the scales to the right of the first mean) is calculated and again the number of structures less and more than this mean in the tail are counted. This is repeated until the $n_{\text {less }} \leq n_{\text {more }}$. The Ht-index is the number of recursions plus one, representing the number of scale interval divisions.

Consider for example, a DPT decomposition consisting of 8 pulses with sizes $1,1,1,1,2,2,6,7$ respectively. The size of the pulse is the number of non-zero entries in the array, referred to as the scale. The mean of these scales is 2.625 , with the values $1,1,1,1,2,2$ being less than this mean and the values 6 and 7 being larger than this mean, so that $75 \%$ are less than the mean and 25\% larger than the mean. This shows that there is a majority (more than $50 \%$ ) of smaller scales. The values smaller than the mean are then discarded and this process repeated for the scale values 6 and 7 . The mean is then 6.5 for which exactly $50 \%$ of the values are smaller and $50 \%$ are larger. The process thus stops and $\mathrm{Ht}$-index is determined as 2.

We propose here to further calculate the $\mathrm{Ht}$-index of the pulses at each majority smaller scale interval until no further majority smaller scale intervals are present, thereby constructing a multilevel $\mathrm{Ht}$-index for BSD that is specifically applicable to textures in images. For the example above this means we calculate the $H t$-index for the values $1,1,1,1,2,2$ obtaining the mean $1 . \dot{3}$. Then $66 . \dot{6} \%$ of the values are less than this mean (the values $1,1,1,1$ ) and $33 . \dot{3} \%$ are larger (the values 2 , 2 ). The process is repeated for the tail values 2,2 . The mean is 2 so there is no majority percentage and the process stops with $h$ obtained as 2 . The multiscale $H t$-index is then represented as $h=2$ at level 1 and $h=2$ at level 2 .

We now turn to point patterns, denoted by $x=\left\{x_{1}, \ldots, x_{n}\right\}$ and position the DPT decomposition as such. Point patterns are well described elsewhere (Illian et al., 2008). An important property of point patterns, which is relevant in our study, is their invariance under rotations. If the distribution of a point pattern is invariant under rotations about the origin, then it is called isotropic, otherwise it is called anisotropic. Anisotropy in point processes may occur as either geometric, which is equivalent to a linear transformation of a stationary and isotropic process, or zonal if it shows increased intensity along directed lines (Rajala et al., 2018). Anisotropy has been studied in some detail. Directional distributions are derived in König and Schmidt (1992), wavelet analysis has been presented in Rosenberg (2004) and Nicolis et al. (2010), tests on the angular directions have been developed in Ohser and Stoyan (1981), Stoyan and Beneš (1991) and Illian et al. (2008), ellipsoids are fitted to Fry plots to test for geometric isotropy in Rajala et al. (2016) and the second-order intensity function has been used in Guan et al. (2006), to list some. Here we focus on multi-level fractal visualisation of rose diagrams for BSD, extracted from multiscale image decompositions.

The point pattern extracted from the DPT is determined as follows, as in Fabris-Rotelli and Stein (2018). Consider a point process $Z(x)$ on $\mathbb{Z}^{2}$. The spatial window is extent of the image, for example, an $N \times M$ image will be modelled on a continuous rectangular widow of size $[0, N] \times[0, M]$. The process $Z(x)$ takes on 1 if there is a pixel belonging to a pulse at position $x$ and 0 otherwise, where position $x$ is the centre of the pixel on the continuous domain. This is illustrated in Fig. 1. The approach of a regular lattice is also to be considered but adds no additional modelling advantages. ${ }^{2}$

\section{Application}

Fig. 2 shows image D15 of the Brodatz texture database, a linear texture. It is used here due to its obvious upward directionality of texture. In addition, it has a stationary structure in that the intensity does not drastically vary over the domain. Non-stationarity would detract from the investigation here on anisotropy. In Fig. 3 the number of pulses $\phi_{n s}$ of the DPT $f=\sum_{n=1}^{N} \sum_{s=1}^{\gamma(n)} \phi_{n s}$ is plotted. The total number of pulses equals 139131 for this texture. The number of pulses $\gamma(n)$ for small $n$ is large and when plotted for all $n$ the plotted values are so close to the $x$-axis, not

\footnotetext{
2 Spatial lattice data typically consists of polygonal areas. When these polygons represent the pixel areas the lattice is referred to as regular.
} 


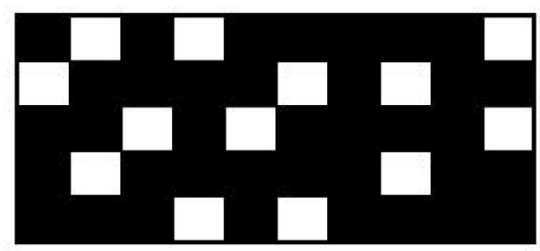

(a)

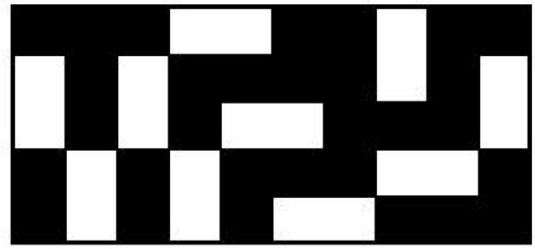

(c)

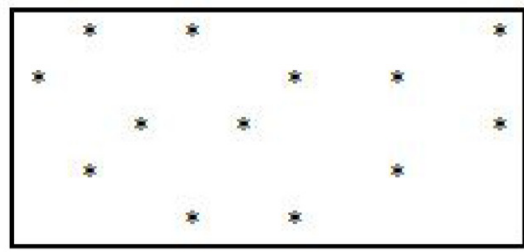

(b)

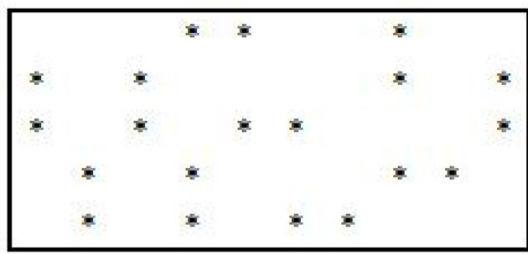

(d)

Fig. 1. (a) Sample of pixels at scale 1 (black indicates background, white indicates foreground); (b) Extracted point pattern for (a); (c) Sample of pixels at scale 2; (d) Extracted point pattern for (c).

discernible as the values decrease from very large to very small at a large pace. In Fig. 3 only scales 1 to 100 are thus shown. The number of pulses $\gamma(n)$ for $n=1$ to 100 is shown in Fig. 3(a), whereas Fig. 3(b) shows the logarithm of both axes, the Richardson plot of Mandelbrot (1967, 1982). This plot is indicative of self-similarity: a perfect straight line shows strict self-similarity and the slope of the line is the fractal dimension. Spatial data are known to not show strict self-similarity, but rather statistical self-similarity with the points around the line rather than on it (Jiang and Yin, 2014). This requires a functional form other than a power function, for example the use of the $H t$-index.

We start by looking at scales $n=1$ to 114 . The reason for choosing 114 will become clear shortly. The point pattern extracted when using pulses at scales $n=1$ to 114 results in 243969 points over a continuous spatial domain $[0,640] \times[0,640]$ (resulting from the image of $640 \times 640$ pixels). The associated image in shown in Fig. 4(a). ${ }^{3}$ This is a partial reconstruction obtained as

$$
f_{\text {rec }}=\sum_{n=n_{1}}^{n_{2}} \sum_{s=1}^{\gamma(n)} \phi_{n s}
$$

for scale interval $\left[n_{1}, n_{2}\right]$. Simple sampling will not be effective for such a large pattern. Decomposition of the point pattern into meaningful scale partitions for sub-analysis proves valuable. We propose the use of the $\mathrm{Ht}$-index in this endeavour.

Fig. 5 graphically illustrates the partition of the large point pattern of image D15 using the Htindex. At first application the number of pulses at each scale $n$ results in recursively calculated mean scales 114, 940, 4886, 17836,49140 and 107423 which have majority of pulses, shown in percentages to the left of the mean in the figure, respectively. The majority percentages are $89 \%$, $86 \%, 81 \%, 78 \%, 70 \%$ and $55 \%$. A high value for $h$ implies a larger complexity. This gives a $H t$-index of $h=7$, indicating a complex structure. The $h=7$ implies that 6 means were calculated resulting in 7 scale intervals. Note the choice of the value of 114 should now be clear as 114 is the first mean calculated. Fig. 4 shows the reconstructed images for each of these scale intervals. We propose to further implement the $\mathrm{Ht}$-index within each of these majority intervals as the size of the point

3 Note: These grey levels are chosen by default for visual purposes when reconstructing the images with less of the original information in order to maximally distinguish between the different areas (pulses) in the reconstruction. They are simply visual grey levels scaled for maximum differentiation. 


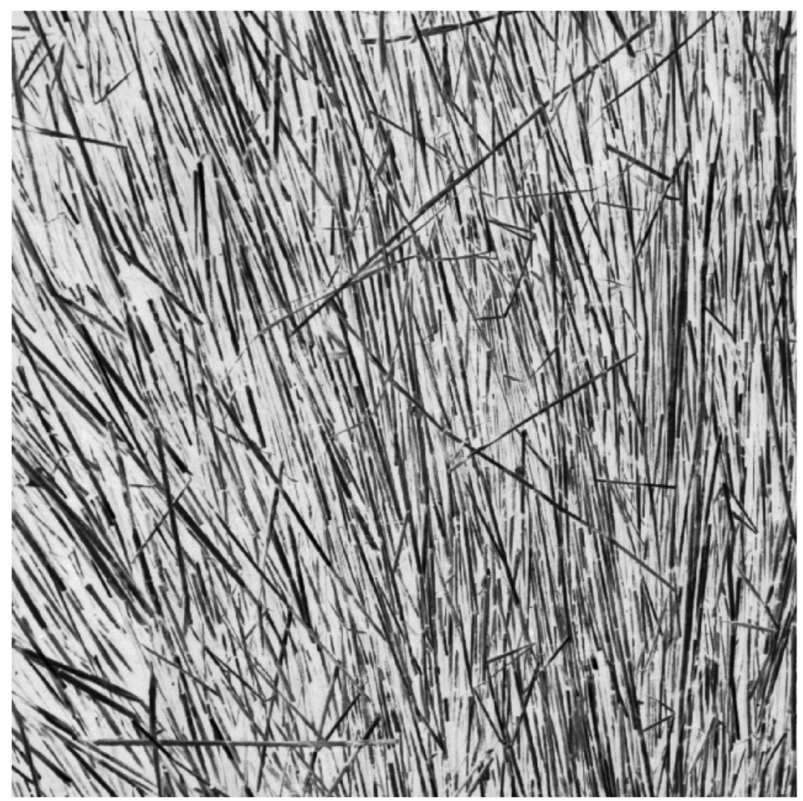

Fig. 2. Image D15 from the Brodatz texture database.

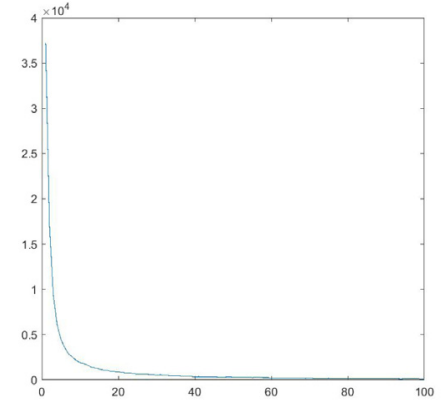

(a) Number of pulses for $n=1$ to 100

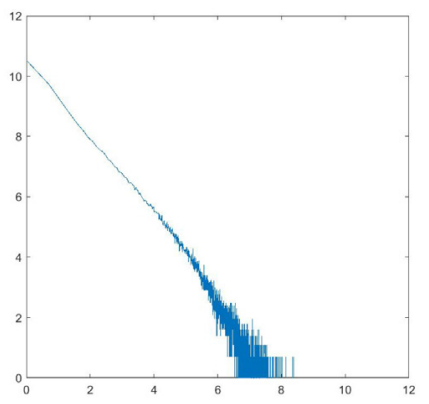

(b) Richardson plot: $\log (n)$ vs $\log (\gamma(n))$

Fig. 3. Number of pulses extracted using the DPT for image D15 of Fig. 2.

patterns in these 6 intervals is still large, see Table 1 . Implementing the $H t$-index on the pulses over scales [1,114] results in means $14,44,71$ and 90 with the majority of pulses to the left of the mean, resulting in $h=5$. The percentages are also shown, as before, in Fig. 5 . This is repeated for scales $[115,940]$ which results in means $265,477,657,774$ and 851 , with $h=6$. The remaining 4 intervals corresponding to means 4886, 17836,49140 and 107423 show no self-similarity, that is, no majority to the left. This second application of the $\mathrm{Ht}$-index within the first scale intervals of $h=7$ is labelled as level 2 in Fig. 5 . Level 3 is the $H t$-index applied to the level 2 intervals. Interval $[1,14]$ has means 3 and 6 , with $h=3$. Interval $[15,44]$ has $h=2$, as does interval $[45,71]$. Interval 


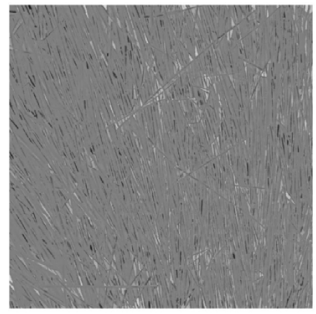

(a) $[1,114]$

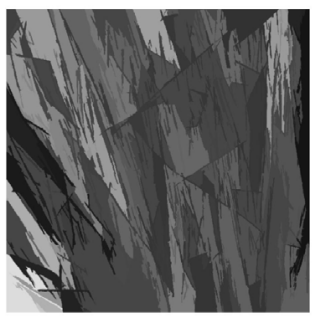

(d) $[4887,17836]$

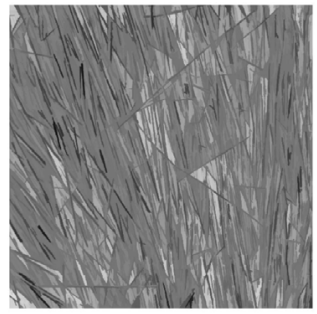

(b) $[115,940]$

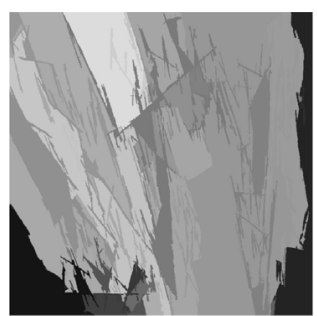

(e) $[17836,49140]$

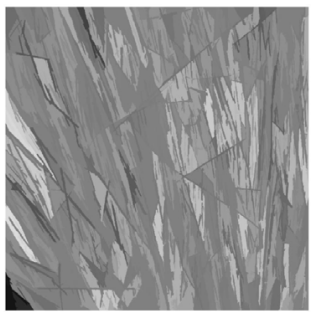

(c) $[941,4886]$

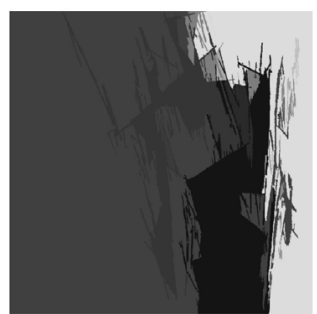

(f) [49141, 107423]

Fig. 4. Partial DPT reconstructions for the scale intervals of level 1 of the multi-level Ht-index using Eq. (1).

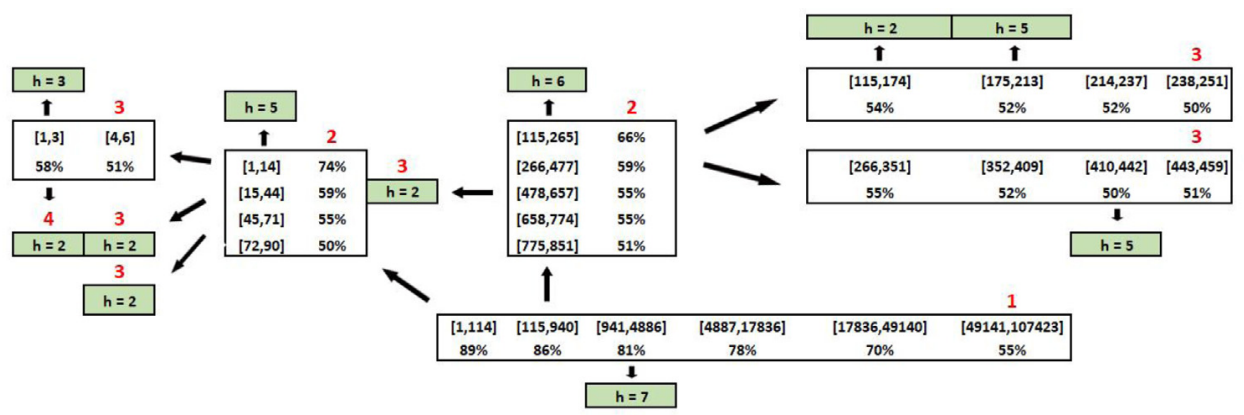

Fig. 5. Multi-level $H t$-index for Brodatz image D15. Scale intervals are group and their $H t$-index, $h$, shown for each group. Levels 1, 2, 3 and 4 indicate the level of the $H t$-index i.e. at what recursion it was calculated, creating a hierarchical structure.

$[115,265]$ has means 174, 213, 237 and 251, with $h=5$. Interval [266, 477] has means 351, 409, 442 and 459 , also with $h=5$. Interval [478,657] has $h=2$. Level 4 is only present for intervals [1, 3] and $[115,174]$ both with $h=2$. We only consider scale intervals for $h>2$ to extract maximum complexity. In Fig. 5 those intervals without an $\mathrm{Ht}$-index show no fractal structure, that is there is no majority pulses to the left of the mean. A full fractal decomposition of the DPT scale-space has been achieved. This is the first mechanism for the DPT enabling an informed partition by scale of the scale-space (see Figs. 6 and 7).

Figs. 8 and 9 show the kernel density fitted intensities of each scale interval for levels 1 to 3 . The intensities for scale [1,114] on level $1,[1,14]$ on level 2 and [1,3] on level 3, the smallest scales at each level, show a strong trend and they capture the complexity of the texture. The associated 
Table 1

Point pattern sizes for each scale interval at levels 1-3.

\begin{tabular}{llllll}
\hline Level 1 & & Level 2 & & Level 3 \\
\hline$[1,114]$ & 243969 & {$[1,14]$} & 133470 & {$[1,3]$} & 74251 \\
{$[115,940]$} & 100 & {$[15,44]$} & 15 & {$[4,6]$} & 4 \\
\hline & & {$[45,71]$} & 44 & {$[115,174]$} & 100 \\
& {$[72,90]$} & 73 & {$[175,213]$} & 100 \\
& & {$[115,265]$} & 100 & {$[214,237]$} & 195 \\
& {$[266,477]$} & 213 & & {$[238,351]$} & 231 \\
& {$[478,657]$} & 477 & & {$[352,409]$} & 353 \\
& {$[658,774]$} & 610 & {$[410,442]$} & 413 \\
& & {$[775,851]$} & 759 & {$[443,459]$} & 376 \\
\hline
\end{tabular}

rose diagrams are shown in the same figure. ${ }^{4}$ The vertical anisotropy is captured in the scales [1, $114],[1,14]$ and $[1,3]$ and more so at level 3 than at level 1 . The image under consideration has mostly vertical directionality but the left of the image can be seen to have some directionality to the North-West. The rose diagrams pick this up in the point pattern extracted from the DPT. This indicates the ability of a multi-level $\mathrm{Ht}$-index decomposition for capturing the scales contributing most significantly to the texture structure. These three scale intervals also have the largest point pattern size as seen in Table 1. The sizes are 243969, 133470 and 74251 respectively. This Ht-index decomposition thus provides an informative way to reduce the data size to the 74251 of scales [1, 3], which captures the anisotropy in a clear manner. The DPT is computationally heavy due to not being parallelisable when implemented (de Lancey and Fabris-Rotelli, 2019). It however provides useful information for the scale information in an image enabling many image processing improved results, see Fabris-Rotelli and Stoltz (2012) for example. The size of the point pattern extracted from the pulses as described earlier is very large resulting in many spatial point pattern analysis tools not being viable for example a Fry plot and model fitting as well as diagnostics (Fabris-Rotelli and Stein, 2018). Having a method to thus reduce the number of pulses of the DPT and the extracted point pattern means that extracting the anisotropy information is at a more manageable data size. Thus the big data and computational issues have been alleviated by analysing the appropriate scales of the DPT. This approach also provides avenues for future techniques employing the DPT pulses when appropriate scale choices should be made. It allows for data specific scale choice instead of ad hoc decisions.

The method of using the DPT to extract pulses from an image and then representing the pulses in a spatial point pattern means that the anisotropy detected is that of the original image process. The origin of the image content is of course continuous from the underlying process, captured into a discretised image. With higher resolution the captured image more accurately represents the true scene. This should always be considered in image analysis as an accuracy level and what results are referred back to the true scene. The image process is presented in an alternative form through a point pattern but the same information is captured. The idea of using the $\mathrm{Ht}$-index is to determine the scales from the multiscale DPT decomposition in which the anisotropy is present. Most of the scale levels are details (at fine scales) or background shading (at coarse scales). We thus harness this multiscale representation using the $\mathrm{Ht}$-index and determine the scales which carry the anisotropy information of the process.

The results on the anisotropic image show that the $H t$-index provides useful breaks for placing different scale intervals into hierarchical levels for optimised spatial analysis of BSD. The anisotropy is captured at other scale intervals as well (see Figs. 8 and 9) but is not as strong. This provides a novel data-driven technique to model using the correct scales of the DPT that has not been applied

\footnotetext{
4 The rose diagram implemented here does not use the point pair distribution. The implementation used is that of $S$ patstat in $R$ and uses the nearest neighbour angles only. It does not thus repeat in the opposite direction producing symmetry. Of course one could take the $n$th nearest neighbours as an alternative, say up to $n=10$ (with $n$ chosen wisely). This was investigated and only adds more smoothing to the rose diagram.
} 


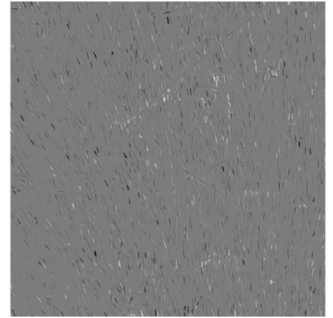

(a) $[15,43]$

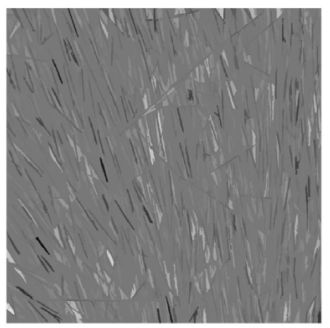

(d) $[115,265]$

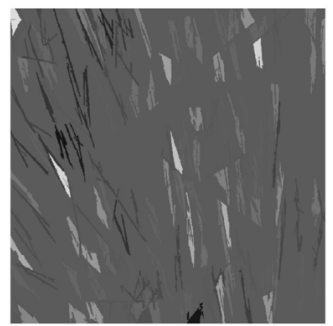

(g) $[658,774]$

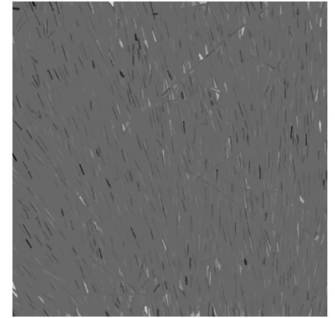

(b) $[44,71]$

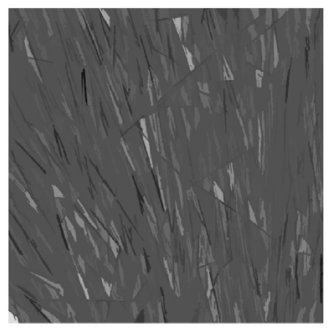

(e) $[266,477]$

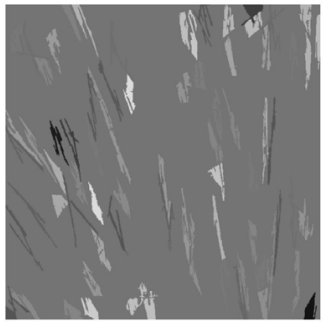

(h) $[775,851]$

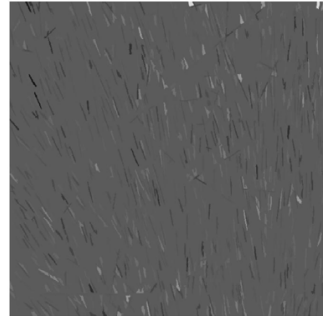

(c) $[72,90]$

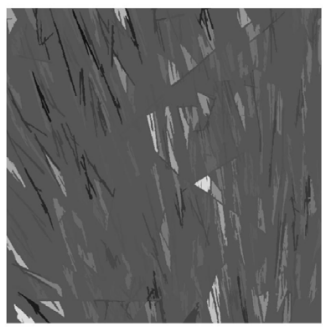

(f) $[478,657]$

Fig. 6. Partial DPT reconstructions for the scale intervals of level 2 of the multi-level $H t$-index.

to images decomposed in a multiscale manner before. Further investigation could delve deeper into the philosophical components of texture such as investigated in Jiang (2015b).

The intensities shown in Figs. 8 and 9 also pick up the heterogeneity of the texture. The three intervals $[1,114],[1,14]$ and $[1,3]$ are those scale intervals containing the heterogeneity. The other intervals show only isolated intensities, not indicative of a pattern. This multi-level $\mathrm{Ht}$-index thus also provides a mechanism for characterising heterogeneity.

To provide a counter example, what we deemed the most isotropic image from the Brodatz texture database was also investigated, image D110, seen in Fig. 10. The Ht-index multiscale method proposed nonetheless still picks up the anisotropy in this image as well. This is due to the natural anisotropic nature of all images. In Hyvärinen et al. (2009, Section 5.7) the anisotropy of natural images is confirmed through measurement of image statistics. This is obvious once one thinks about it. A natural image must have rotational variation to express its content, even with repetition present. Thus, a true isotropic image can only be simulated. 


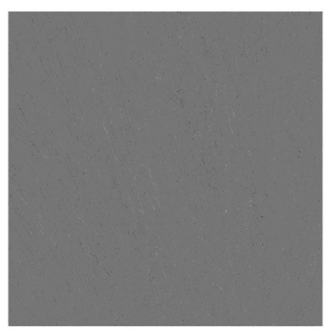

(a) $[1,3]$

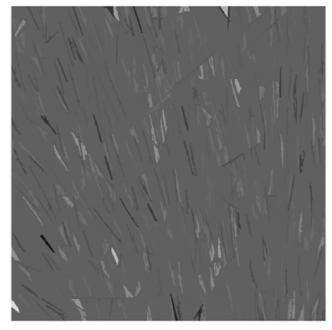

(d) $[175,213]$

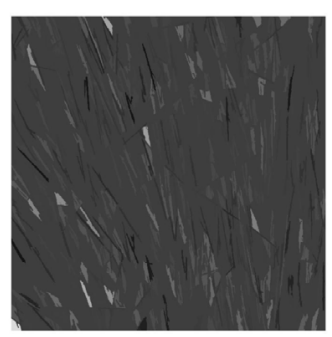

(g) $[266,351]$

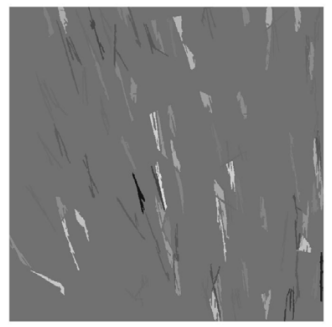

(j) $[443,459]$

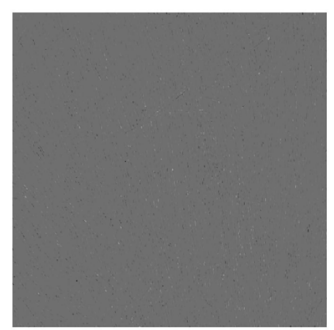

(b) $[4,6]$

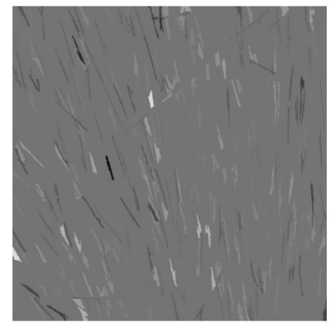

(e) $[214,237]$

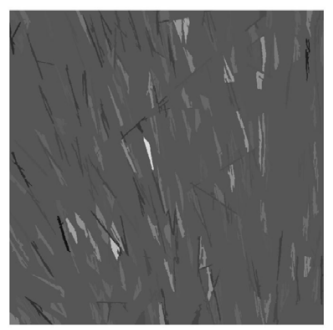

(h) $[352,409]$

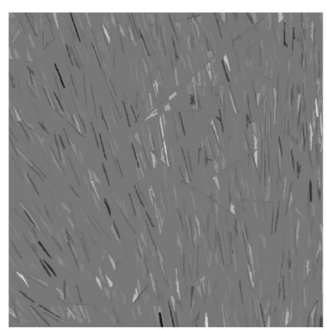

(c) $[115,174]$

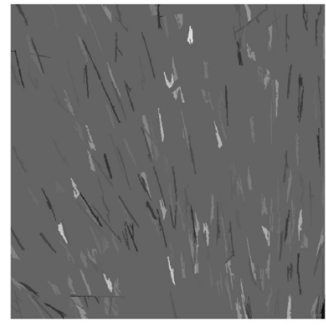

(f) $[238,351]$

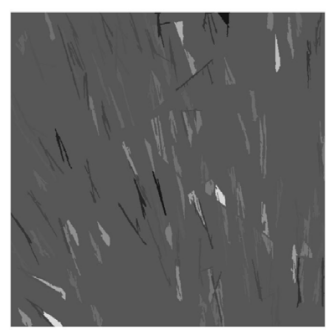

(i) $[410,442]$

Fig. 7. Partial DPT reconstructions for the scale intervals of level 3 of the multi-level $\mathrm{Ht}$-index. 
LEVEL 1

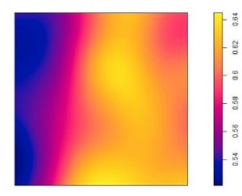

(a) $[1,114$

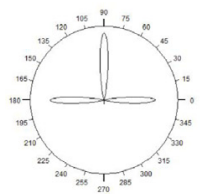

LEVEL 2

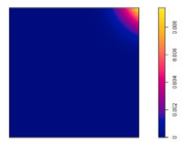

(b) $[115,940]$

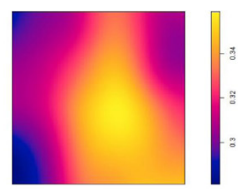

(c) $[1,14]$

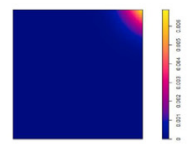

(f) $[72,90]$

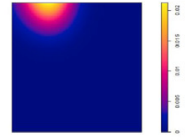

(i) $[478,657]$
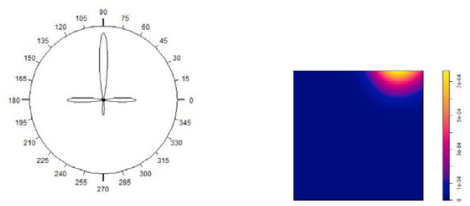

(d) $[15,44]$

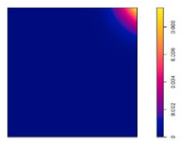

(g) $[115,265]$

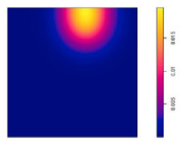

(j) $[658,774]$

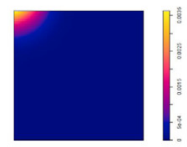

(e) $[45,71]$

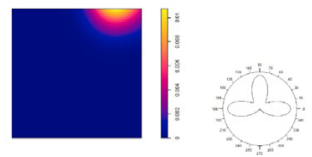

(h) $[266,477]$

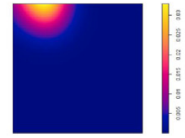

(k) $[775,851]$

Fig. 8. Kernel density plots of the intensities and rose diagrams for the associated point patterns at each intervals for levels 1-2. Plots (a) and (c) are shown larger for details.

\section{Discussion}

This paper focused on anisotropy, whereas, more complex spatial modelling can also be investigated, for example fitting cluster models at each level enabling parallelisation of spatial data. In addition, multiple bands and spatio-temporal data should also be investigated for a fractal decomposition.

There have been two suggested extensions to Jiang's $H t$-index: the $C R G$-index (Gao et al., 2016a) and the ratio of areas $(R A)$ (Gao et al., 2016b). The CRG index is said to be a more sensitive $H t$-index using adaptive breaks, and the $R A$ captures more heterogeneity. The implications of these to the DPT may yield further insights in future research.

Image D15 of the Brodatz texture database was chosen as it does not exhibit any obvious nonstationarity. Future analysis should determine if the multi-level $\mathrm{Ht}$-index proposed can pick up non-stationarity. The Second Law of Geography states that geographic events are more likely to occur in some locations than at other locations, that is, spatial non-stationarity. There is also not something like an average location (Anselin, 1989; Goodchild, 2004). Non-stationarity should be 


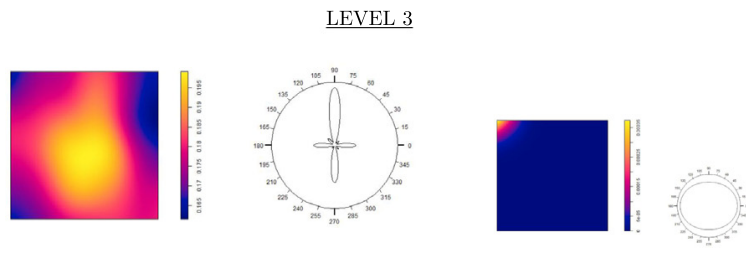

(a) $[1,3]$

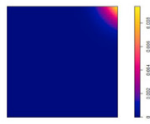

(c) $[115,174]$

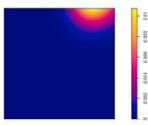

(g) $[266,351]$

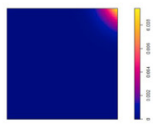

(d) $[175,213$

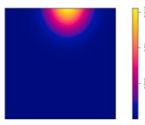

(h) $[352,409$

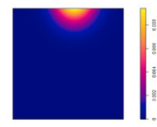

(e) $[214,237]$

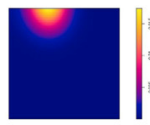

(i) $[410,442]$

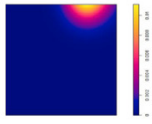

(f) $[238,251]$

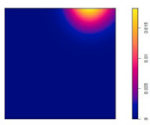

(j) $[443,459]$

Fig. 9. Kernel density plots of the intensities and rose diagrams for the associated point patterns at each intervals for levels 3. Plot (a) is shown larger for details.

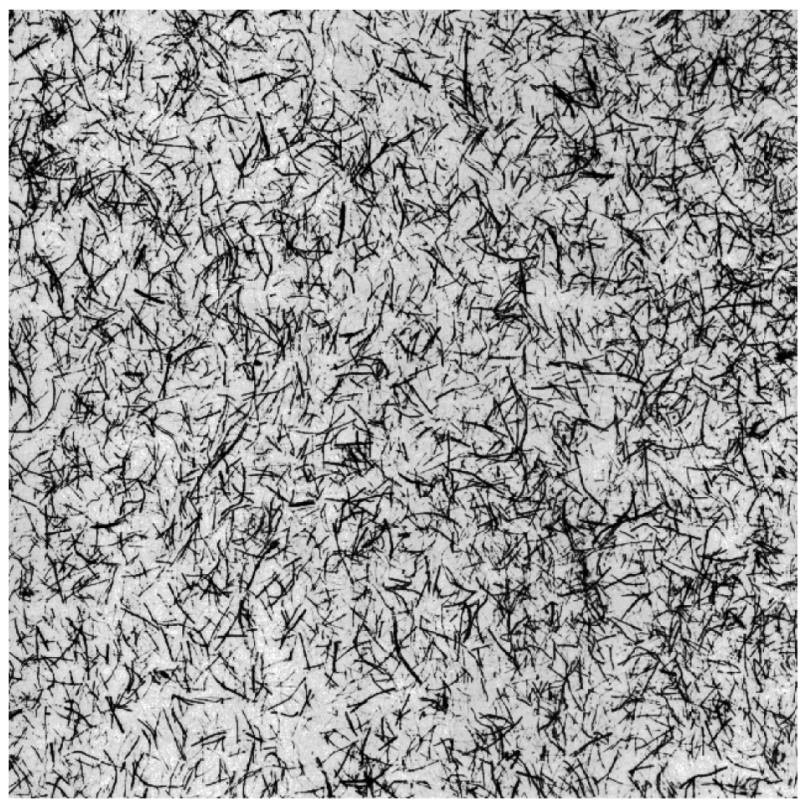

Fig. 10. Image D110 from the Brodatz texture database.

determined for data being considered. Interestingly, the $\mathrm{Ht}$-index has been used to capture nonstationarity in spatial data as well as (Ma et al., 2015) and was able to formulate non-stationarity 
as a scaling law (Jiang, 2015a). Modelling of this specific texture using a line process should also provide interesting results and we plan to do this in a future study.

The presence of noise in an image should not be disregarded. Noise (if additive and not multiplicative) will simply add more small structures, thus increasing the Ht-index. Any anisotropy identification in images should involve a noise removal step, as do most image processing tasks. An interesting phenomenon may occur in the presence of multiplicative noise, where the noise may be part of the signal as in radar images. But here again, this more structural form of noise should be dealt with before testing for anisotropy. A simulation study on various noise types added will yield interesting results in the future.

\section{Conclusion}

This study showed that the $H t$-index is an efficient and effective visualisation tool to analyse anisotropic point patterns obtained from images as a specific form of spatial big data. We have shown this for a point pattern extracted from a texture image process, using the extracted fractal levels for anisotropy analysis. Applying the $\mathrm{Ht}$-index at multiple levels provides useful breaks for optimised spatial analysis. We found that the multi-level Ht-index is an important method for anisotropy analysis. It allowed us to deal with spatial big data in a knowledgeable, data-driven way, and it captures the inherent hierarchy of spatial features. We foresee that this will be significant as well for use in statistical mapping and cognitive mapping. In future the simulation of directional self-similar images, such as in Roux et al. (2013), will provide an extension to this study. In addition, a study of simulated isotropic and anisotropic images, similar to the approach in Richard (2016), will reveal further insight to this proposed new multiscale Ht-index.

\section{Acknowledgements}

Funding from the University of Pretoria's Vice-Principal Congress Funding and Statomet from the Department of Statistics, University of Pretoria is acknowledged for this research.

\section{References}

Al-Saidi, N., Abdul-Wahed, H., 2018. Classification of remote sensing images via fractal discriptores. In: 2018 International Conference on Advance of Sustainable Engineering and Its Application. ICASEA, IEEE, pp. 99-104.

Anguelov, R., Fabris-Rotelli, I., 2010. LULU operators and discrete pulse transform for multidimensional arrays. IEEE Trans. Image Process. 19 (11), 3012-3023.

Anselin, L., 1989. What is Special About Spatial Data? Alternative Perspectives on Spatial Data Analysis (89-4). Technical Report, National Center for Geographic Information and Analysis, Santa Barbara, CA.

Avadhanam, N., Mitra, S., 1994. Analysis of texture images using robust fractal description. In: Proceedings of the IEEE Southwest Symposium on Image Analysis and Interpretation. IEEE, pp. 1-6.

Bai, Z., Zhang, W., Zhou, M., Bai, J., 2011. Algorithm designed for image inpainting based on decomposition and fractal. In: Proceedings of 2011 International Conference on Electronic \& Mechanical Engineering and Information Technology, Vol. 3. IEEE, pp. 1552-1555.

Berizzi, F., Dell'Acqua, F., Gamba, P., Garzelli, A., Martorella, M., 2001. On the fractal behavior of SAR images of ocean sea surface. In: IGARSS 2001. Scanning the Present and Resolving the Future. Proceedings. IEEE 2001 International Geoscience and Remote Sensing Symposium (Cat. No. 01CH37217), Vol. 4. IEEE, pp. 1729-1731.

Chen, S., Keller, J., Crownover, R., 1993. On the calculation of fractal features from images. IEEE Trans. Pattern Anal. Mach. Intell. 15 (10), 1087-1090.

Chen, X., Vo, H., Aji, A., Wang, F., 2014. High performance integrated spatial big data analytics. In: 3rd ACM Sigspatial International Workshop on Analytics for Big Geospatial Data, pp. 11-14.

Chenoweth, D., Cooper, B., Selvage, J., 1995. Aerial image analysis using fractal-based models. In: 1995 IEEE Aerospace Applications Conference. Proceedings, Vol. 2. IEEE, pp. 277-285.

Costa, A., Humpire-Mamani, G., Traina, A., 2012. An efficient algorithm for fractal analysis of textures. In: 2012 25th SIBGRAPI Conference on Graphics, Patterns and Images. IEEE, pp. 39-46.

Cugler, D., Oliver, D., Evans, M., Shekhar, S., Medeiros, C., 2013. Spatial big data: platforms, analytics, and science. GeoJournal.

de Lancey, M., Fabris-Rotelli, I., 2019. Effective graph sampling of a nonlinear image transform. In: Proceedings of FAIR 2019, Vol. 2540, Cape Town, pp. 185 - 195, http://ceur-ws.org/Vol-2540/.

Di Martino, G., Iodice, A., Riccio, D., Ruello, G., Zinno, I., 2010. Fractal based filtering of SAR images. In: 2010 IEEE International Geoscience and Remote Sensing Symposium. IEEE, pp. 2984-2987. 
Dolez, B., Vincent, N., 2007. Sample selection in textured images. In: 2007 IEEE International Conference on Image Processing, Vol. 2. IEEE, pp. II-221.

Evans, M., Oliver, D., Yang, K., Zhou, X., Ali, R., Shekhar, S., 2018. Cyber GIS for geospatial discovery and innovation. In: Wang, S., Goodchild, M. (Eds.), Springer, Dortrecht, pp. 143-170.

Evans, M., Oliver, D., Zhou, X., Shekhar, S., 2014. Big data: Techniques and technologies in geoinformatics. In: Karimi, H. (Ed.), CRC Press.

Fabris-Rotelli, I., Stein, A., 2018. Inhomogeneous spatial modelling of DPT pulses for marine images. Spat. Stat. 28, 257-270.

Fabris-Rotelli, I., Stoltz, G., 2012. On the leakage problem with the Discrete Pulse Transform decomposition. In: de Waal, A. (Ed.), Proceedings of the 23rd Annual Symposium of the Pattern Recognition Association of South Africa, pp. 179-186, ISBN: 978-0-620-54601-0.

Fazel-Rezai, R., Kinsner, W., 1999. Texture analysis and segmentation of images using fractals. In: Engineering Solutions for the Next Millennium. 1999 IEEE Canadian Conference on Electrical and Computer Engineering (Cat. No. 99TH8411), Vol. 2. IEEE, pp. 786-791.

Gao, P., Liu, Z., Tian, K., Liu, G., 2016b. Characterizing traffic conditions from the perspective of spatial-temporal heterogeneity. ISPRS Int. J. Geo-Inf. 5 (3), 34.

Gao, P., Liu, Z., Xie, M., Tian, K., Liu, G., 2016a. CRG index: A more sensitive ht-index for enabling dynamic views of geographic features. Prof. Geogr. 68 (4), 533-545.

Ghazel, M., Freeman, G., Vrscay, E., 2003. Fractal image denoising. IEEE Trans. Image Process. 12 (12), 1560-1578.

Goodchild, M., 2004. Giscience, geography, form, and process. Ann. Assoc. Am. Geogr. 94 (4), 709-714.

Goodchild, M., 2013. The quality of big(geo)data. Dialogues Hum. Geogr. 3 (3).

Guan, Y., Sherman, M., Calvin, J., 2006. Assessing isotropy for spatial point processes. Biometrics 62 (1), 119-125.

Hamano, T., Tokuda, K., Kaneko, M., 1996. Image restoration based on estimation of fractal structure. In: Proceedings of Digital Processing Applications, Vol. 1. TENCON'96, IEEE, pp. 311-316.

Hong, S., Huidong, D., 2012. Fractal dimension applied in texture feature extraction in x-ray chest image retrieval. In: 2012 IEEE International Conference on Information and Automation. IEEE, pp. 841-845.

Hyvärinen, A., Hurri, J., Hoyer, P., 2009. Natural Image Statistics: A Probabilistic Approach to Early Computational Vision. Springer Science and Business Media.

Ida, T., Sambonsugi, Y., 1998. Image segmentation and contour detection using fractal coding. IEEE Trans. Circuits Syst. Video Technol. 8 (8), 968-975.

Illian, J., Penttinen, A., Stoyan, H., Stoyan, D., 2008. Statistical Analysis and Modelling of Spatial Point Patterns, Vol. 70. John Wiley \& Sons.

Ismail, I., Hamdy, A., Frig, R., 2010. Studying the effect of down sampling and spatial interpolation on fractal image compression. In: The 2010 International Conference on Computer Engineering \& Systems. IEEE, pp. 355-360.

Jardak, C., Mahonen, P., Riihijarvi, J., 2014. Spatial big data and wireless networks: experiences, applications, and research challenges. IEEE Network 26-31.

Jenks, G.F., 1963. Generalization in statistical mapping. Ann. Assoc. Am. Geogr. 53 (1), 15-26.

Jiang, J., 1995. Image compression with fractals. In: IEE Colloquium on Fractals in Signal and Image Processing, London, UK. IET, pp. $7 / 1-7 / 3$.

Jiang, B., 2013. Head/tail breaks: A new classification scheme for data with a heavy-tailed distribution. Prof. Geogr. 65 (3), 482-494.

Jiang, B., 2015a. Geospatial analysis requires a different way of thinking: The problem of spatial heterogeneity. GeoJournal 80 (1), 1-13.

Jiang, B., 2015b. Wholeness as a hierarchical graph to capture the nature of space. Int. J. Geogr. Inf. Sci. 29 (9), $1632-1648$.

Jiang, B., Liu, X., Jia, T., 2013. Scaling of geographic space as a universal rule for map generalization. Ann. Assoc. Am. Geogr. 103 (4), 844-855.

Jiang, B., Miao, Y., 2015. The evolution of natural cities from the perspective of location-based social media. Prof. Geogr. 67 (2), 295-306.

Jiang, B., Sui, D., 2014. A new kind of beauty out of the underlying scaling of geographic space. Prof. Geogr. 66 (4), $676-686$.

Jiang, B., Yin, J., 2014. Ht-index for quantifying the fractal or scaling structure of geographic features. Ann. Assoc. Am. Geogr. 104 (3), 530-540.

Kisan, S., Mishra, S., Mishra, D., 2016. A novel method to estimate fractal dimension of color images. In: 2016 11th International Conference on Industrial and Information Systems. ICIIS, IEEE, pp. 692-697.

König, D., Schmidt, V., 1992. Directional distributions for multi-dimensional random point processes. Stoch. Models 8 (4), 617-636.

Kumar, P., 1995. A wavelet based methodology for scale-space anisotropic analysis. Geophys. Res. Lett. 22 (20).

Lam, N., 1990. Description and measurement of Landsat TM images using fractals. Photogramm. Eng. Remote Sens. 56 (2), 187-195.

Lam, A., Li, Q., 2010. Fractal analysis and multifractal spectra for the images. In: 2010 International Symposium on Computer, Communication, Control and Automation, Vol. 2. 3CA, IEEE, pp. 530-533.

Li, S., Dragicevic, S., Castro, F., Sester, M., Winter, S., Coltekin, A., Pettit, C., Jiang, B., Haworth, J., Stein, A., Cheng, T., 2016. Geospatial big data handling theory and methods: A review and research challenges. ISPRS J. Photogramm. Remote Sens. 115, 119-133.

Li, X., Li, W., Anselin, L., Rey, S., Koschinsky, J., 2014. A MapReduce algorithm to create contiguity weights for spatial analysis of big data. In: Proceedings of the 3rd ACM SIGSPATIAL International Workshop on Analytics for Big Geospatial Data, pp. 50-53. 
Liu, S., 2008. An improved differential box-counting approach to compute fractal dimension of gray-level image. In: 2008 International Symposium on Information Science and Engineering, Vol. 1. IEEE, pp. 303-306.

Lopez, D., Gunasekaran, N., Murugan, B., Kaur, H., Abbas, K., 2014. Spatial big data analytics of Influenza epidemic in Vellore, India. In: 2014 IEEE Conference on Big Data, pp. 19-24.

Ma, D., Sandberg, M., Jiang, B., 2015. Characterizing the heterogeneity of the openstreetmap data and community. ISPRS Int. J. Geo-Inf. 4 (2), 535-550.

Malviya, A., 2008. Fractal based spatial domain techniques for image de-noising. In: 2008 International Conference on Audio, Language and Image Processing. IEEE, pp. 1511-1516.

Mandelbrot, B., 1967. How long is the coast of Britain? statistical self-similarity and fractional dimension. Science 156 (3775), 636-638.

Mandelbrot, B., 1982. The Fractal Geometry of Nature, San Francisco, CA.

Manogaran, G., Lopez, D., 2018. Spatial cumulative sum algorithm with big data analytics for climate change detection. Comput. Electr. Eng. 65, 207-221.

Melnikov, G., 2007. The fractal method of the image coding. In: 2007 Siberian Conference on Control and Communications. IEEE, pp. 153-157.

Miller, H., Goodchild, M., 2015. Data driven geography. GeoJournal 80 (4), 449-461.

Miya, J., Aghagotza, A., 2004. Edge detection using directional wavelet transform. In: Proceedings of the 12th IEEE Maditerranean Electrotechnical Conference, Dubronik, Canada.

Nayak, S., Ranganath, A., Mishra, J., 2015. Analysing fractal dimension of color images. In: 2015 International Conference on Computational Intelligence and Networks. IEEE, pp. 156-159.

Nicolis, O., Mateu, J., D’Ercole, R., 2010. Testing for anisotropy in spatial point processes. In: Gonzalez-Manteiga, et al. (Eds.), Proc 5th Int Workshop on Spatio-Temporal Modelling. METMA5, Unidixital, ISBN: 978-840934272.

Nicolis, O., Raminez-Cabo, P., Vidakovic, B., 2011. 2D Wavelet based spectra with applications. Comput. Statist. Data Anal. 55 (1), 738-751.

Ohser, J., Stoyan, D., 1981. On the second-order and orientation analysis of planar stationary point processes. Biom. J. 23 (6), 523-533.

Po, D., Do, M., 2006. Directional multiscale modeling of images using the contourlet transfrom. IEEE Trans. Image Process. 15 (6), 1610-1620.

Priya, E., Srinivasan, S., Ramakrishnan, S., 2011. Differentiation of digital TB images using multi-fractal analysis. In: 2011 24th Canadian Conference on Electrical and Computer Engineering. CCECE, IEEE, pp. 1431-1434.

Qi, D., Jin, X., Wu, H., 2009. Application of multi-scale fractal feature in defects detection of log X-ray image. In: 2009 WRI Global Congress on Intelligent Systems, Vol. 3. IEEE, pp. 155-159.

Rajala, T., Redenbach, C., Särkkä, A., Sormani, M., 2018. A review on anisotropy analysis of spatial point patterns. Spat. Stat. 28, 141-168.

Rajala, T., Särkkä, A., Redenbach, C., Sormani, M., 2016. Estimating geometric anisotropy in spatial point patterns. Spat. Stat. $15,100-114$.

Ratcliffe, J., 2004. Geocoding crime and a first estimate of a minimum acceptable hit rate. Int. J. Geogr. Inf. Sci. 18 (1), 61-72.

Riccio, D., Di Martino, G., Iodice, A., Ruello, G., Zinno, I., 2014. Fractal dimension images from SAR images. In: 2014 IEEE International Conference on Image Processing. ICIP, IEEE, pp. 106-110.

Richard, F., 2016. Tests of isotropy for rough texturesof trended images. Statist. Sinica 26 (3), 1279-1304.

Rigaut, J., Schoevaert-Brossault, D., Downs, A., Landini, G., 1998. Asymptotic fractals in the context of grey-scale images. J. Microsc. 189 (Pt 1), 57-63.

Rosen, D., 1995. Fractal features of the digital measurement of length and area. unpublished.

Rosenberg, M., 2004. Wavelet analysis for detecting anisotropy in point patterns. J. Veg. Sci. 15 (2), 277-284.

Roux, S., Clausel, M., Vedel, B., Jaffard, S., Abry, P., 2013. Self-similar anisotropic texture analysis: the hyperbolic wavelet transform contribution. IEEE Trans. Image Process. 22 (11), 4353-4363.

Sawada, K., Nagai, S.-Y., Nakamura, E., 2001. Fractal image coding combined with subband decomposition. In: ICECS 2001. 8th IEEE International Conference on Electronics, Circuits and Systems, Vol. 3. IEEE, pp. 1347-1350.

Shekhar, S., Evans, M., Kang, J., Mohan, P., 2011. Identifying patterns in spatial information: a survey of methods. Wiley Interdiscip. Rev.: Data Min. Knowl. Discov. 1 (3), 193-214.

Shekhar, Z., Jiang, S., 2017. Spatial Big Data Science. Springer.

Shi, Z., Yao, S., Li, B., Cao, Q., 2008. A novel image interpolation technique based on fractal theory. In: 2008 International Conference on Computer Science and Information Technology. IEEE, pp. 472-475.

Shih, A.-Z., 2008. An examination of fractal dimension approach of image classification. In: 2008 International Conference on Machine Learning and Cybernetics, Vol. 6. IEEE, pp. 3156-3160.

Stoyan, D., Beneš, V., 1991. Anisotropy analysis for particle systems. J. Microsc. 164 (2), 159-168.

Tang, M., Wang, H., 2006. Feature analysis of brain MRI images based on fractal dimension. In: 2005 IEEE Engineering in Medicine and Biology 27th Annual Conference. IEEE, pp. 3245-3248.

Vatsavai, R., Chandola, V., Klasky, S., Ganguly, A., Stefanidis, A., Shekhar, S., 2012. Spatiotemporal data mining in the era of big spatial data: algorithms and applications. In: ACM Conference SigSpatial Big Spatial.

Wang, J., Liu, Y., Wei, P., Tian, Z., Li, Y., Zheng, N., 2011. Fractal image coding using SSIM. In: 2011 18th IEEE International Conference on Image Processing. IEEE, pp. 241-244.

Wang, S., Zhang, Y., Lu, H., Wang, E., Yun, W., Cai, W., 2017. Geospatial big data analytics engine for Spark. In: ACM Conference SigSpatial Big Spatial.

Wee, Y., Shin, H., 2010. A novel fast fractal super resolution technique. IEEE Trans. Consum. Electron. 56 (3), $1537-1541$. 
Xiu-hong, Y., Bao-long, G., 2009. A novel non-local image inpainting algorithm: Fractal-based image inpainting. In: 2009 Second International Workshop on Computer Science and Engineering, Vol. 2. IEEE, pp. 26-30.

Yu, J., Wu, J., Sarwat, M., 2016. A demonstration of GeoSpark: A cluster computing framework for processing big spatial data. In: ICDE 2016 IEEE Conference.

Zhang, L., Chen, J., Qiu, B., 2016a. Region of interest extraction in remote sensing images by saliency analysis with normal directional lifting wavelet transform. Neurocomputing 179, 186-201.

Zhang, G., Huang, Q., Zhu, A.-X., Heel, J., 2016b. Enabling point pattern analysis on spatial big data using cloud computing: optimising and accelrating Ripley's K-function. Int. J. Geogr. Inf. Sci. 30 (11), 2230-2252.

Zhang, G., Zhu, A.-X., Huang, Q., 2017. A GPU-accelerated adaptive kernel density estimation approach for efficient point pattern analysis on spatial big data. Int. J. Geogr. Inf. Sci. 31 (10), 2068-2097.

Zhao, E., Liu, D., 2005. Fractal image compression methods: A review. In: Third International Conference on Information Technology and Applications, Vol. 1. ICITA'05, IEEE, pp. 756-759.

Zhu, L., Yang, J., 2010. Fast multi-spectral image coding algorithm based on fractal. In: 2010 International Symposium on Intelligence Information Processing and Trusted Computing. IEEE, pp. 446-449. 